\title{
Leukocyte telomere length and renal cell carcinoma survival in two studies
}

Catherine L Callahan ${ }^{\star}, 1$, Kendra Schwartz ${ }^{2}$, Julie J Ruterbusch ${ }^{2}$, Brian Shuch ${ }^{3}$, Barry I Graubard ${ }^{4}$, Qing Lan ${ }^{1}$, Richard Cawthon ${ }^{5}$, Andrea A Baccarelli, Wong-Ho Chow ${ }^{7}$, Nathaniel Rothman ${ }^{1}$, Jonathan N Hofmann ${ }^{1,8}$ and Mark P Purdue ${ }^{1,8}$

${ }^{1}$ Occupational and Environmental Epidemiology Branch, Division of Cancer Epidemiology and Genetics, National Cancer Institute, 9609 Medical Center Dr., Rockville, MD 20850, USA; ${ }^{2}$ Department of Family Medicine and Public Health Sciences, Karmanos Cancer Institute, Wayne State University, 3939 Woodward Ave., Detroit, MI 48201, USA; ${ }^{3}$ Department of Urology, Yale School of Medicine, PO Box 208058, New Haven, CT 06520, USA; ${ }^{4}$ Biostatistics Branch, Division of Cancer Epidemiology and Genetics, National Cancer Institute, 9609 Medical Center Dr., Rockville, MD 20850, USA; ${ }^{5}$ Department of Human Genetics, University of Utah, 15 N 2030 E, Salt Lake City, UT 84112, USA; ' Laboratory of Environmental Precision Biosciences, Mailman School of Public Health, Columbia University, 722 West 168th St., New York, NY 10032, USA and 'Department of Epidemiology, Division of OVP, Cancer Prevention and Population Sciences, The University of Texas MD Anderson Cancer Center, Unit 1340 PO Box 301439, Houston, TX 77230-1439, USA

Background: Leukocyte telomere length (LTL) is a potential biomarker of cancer prognosis; however, evidence for renal cell carcinoma $(\mathrm{RCC})$ is inconsistent.

Methods: We investigated LTL and RCC-specific survival among 684 cases from the US kidney cancer study (USKC) and 241 cases from the prostate, lung, colorectal, and ovarian cancer screening trial (PLCO). Leukocyte telomere length was measured by quantitative polymerase chain reaction, and hazard ratios (HRs) and $95 \%$ confidence intervals (Cls) computed using multivariable Cox models.

Results: Short LTL was associated with poorer disease-specific survival in both USKC (lowest vs highest quartile: HR: 2.3, 95\% Cl: 1.2-4.4; $P$ for trend $=0.02$ ) and PLCO (HR: 2.4, 95\% Cl: 1.0-5.4; $P=0.04$ ). Among USKC cases, the association was strongest for stage-I RCC (HR: 5.5, 95\% Cl: 1.6-19.0; $P=0.006)$.

Conclusions: Our findings suggest that shorter LTL is an independent marker of poor RCC prognosis, particularly for stage-I disease.

Telomeres are tandem nucleotide repeats at the ends of chromosomes essential for maintenance of chromosome stability. Leukocyte telomere length (LTL) has been suggested as a biomarker of cancer prognosis (Zhang et al, 2015). A recent meta-analysis found short LTL to be associated with poorer overall cancer survival, although substantial heterogeneity was apparent across cancer types (Zhang et al, 2015). In the case of renal cell carcinoma (RCC), the most fatal urologic malignancy, two small studies have investigated LTL and survival, with conflicting findings. A study of 83 clear cell RCC cases, using blood collected after diagnosis, found longer LTL to be associated with poorer survival (Svenson et al, 2009). Conversely, in a study of 59 kidney cancer cases from a population cohort with pre-diagnostic samples, shorter LTL was non-significantly associated with worse survival (Weischer et al, 2013).

\footnotetext{
*Correspondence: Dr CL Callahan; E-mail: Catherine.callahan@nih.gov

${ }^{8}$ These authors contributed equally to this work.
}

Received 14 April 2017; revised 14 June 2017; accepted 30 June 2017; published online 25 July 2017

(C) 2017 Cancer Research UK. All rights reserved 0007-0920/17 
To clarify the relationship between LTL and RCC prognosis, we conducted an investigation among 684 cases from a US casecontrol study and 241 cases from the prostate, lung, colorectal and ovarian (PLCO) cancer screening trial.

\section{MATERIALS AND METHODS}

Methods of both studies have been described previously (Hofmann et al, 2011, 2013). The US Kidney Cancer Study (USKC) is a population-based case-control study of Caucasians and African Americans conducted in Detroit, MI and Chicago, IL. In Detroit, potentially eligible cases diagnosed between 1 February 2002 and 31 July 2007 were identified through the Metropolitan Detroit Cancer Surveillance System, a member of the NCI Surveillance, Epidemiology, and End Results (SEER) Program. The median time between diagnosis and blood sample collection was approximately 4 months (range, 1-47 months). Deoxyribonucleic acid (DNA) was extracted from whole blood or buffy coat using Qiagen kits (Qiagen Inc., Valencia, CA, USA), and relative LTL was measured by quantitative PCR (Hofmann et al, 2011). Telomere repeat (T) and single gene (S) copy numbers were measured in each sample and adjusted in comparison to standard reference DNA; the standardised T/S ratio characterises relative telomere length. Blinded duplicate samples from 59 participants were interspersed among test samples for quality control; the resulting data suggested high assay reproducibility, with a coefficient of variation (CV) of 9.9\%. The mean T/S ratio among controls was 0.87 (standard deviation (s.d.) 0.20 ). The analyses reported herein are restricted to Detroit cases, for whom vital status and underlying cause of death had been ascertained through 31 December 2012 via SEER linkage (Schwartz et al, 2016).

Prostate, lung, colorectal, and ovarian cancer screening trial is a large multi-centre cancer screening trial, the methods of which have been described (Hayes et al, 2000). Leukocyte telomere length measurements were performed using pre-diagnostic specimens from 241 RCC cases who participated in PLCO as part of a previous nested case-control study (Hofmann et al, 2013). Deoxyribonucleic acid was extracted from buffy coat using Qiagen kits $(n=209)$ or was previously extracted $(n=32)$. Leukocyte telomere length was measured using monochrome multiplex PCR. The CV from 36 blinded quality control samples distributed evenly across nine plates was 5.9\%. The mean T/S ratio among controls was 1.44 (s.d.: 0.89). Cases were followed for vital status and underlying cause of death via linkage with the National Death Index through 31 December 2008.

Leukocyte telomere length was measured in different laboratories using different standard reference DNA. Thus, the two studies were analysed separately, with categorisations of LTL based on study-specific quartiles. We used multivariable Cox proportional hazards models to compute hazard ratios (HRs) and 95\% confidence intervals (CIs) relating disease-specific survival to categories of LTL while adjusting for age, sex, race, study center (PLCO only), body mass index, smoking status, hypertension, diabetes, source of DNA, and education. Data from the USKC study, which unlike PLCO had collected information on tumour characteristics, were further adjusted for histology, stage, treatment, grade, and tumour size. All analyses were conducted using SAS version 9.3 (SAS Institute, Inc., Cary, NC, USA). The proportional hazards assumption was tested using an interaction term for LTL and survival time, which was not significant. Studyspecific results were combined by meta-analysis using a random effects model.

Both studies were approved by Institutional Review Boards at collaborating institutions, and written informed consent was obtained from all participants.

\section{RESULTS}

Characteristics of cases are presented in Table 1. Ninety-six and 62 RCC-related deaths were ascertained among USKC and PLCO cases, respectively. The study-specific associations between LTL and RCC survival are summarised in Table 2. Shorter LTL was associated with poorer disease-specific survival in both USKC (shortest $v s$ longest quartile: HR: $2.3,95 \% \mathrm{CI}: 1.2-4.4 ; P_{\text {trend }}=0.02$ ) and PLCO (HR: 2.4, 95\% CI: 1.0-5.4; $\left.P_{\text {trend }}=0.04\right)$. The association between LTL and RCC survival was in the same direction when stratified by median time from blood collection to diagnosis, but a statistically significant trend was only observed among cases diagnosed $>6.5$ years after blood collection (Table 2). Leukocyte telomere length was not associated with death from other causes in either study (USKC, HR: 0.8, 95\% CI: 0.4-1.4; $P_{\text {trend }}=0.91 ;$ PLCO, HR: $1.4,95 \%$ CI: $0.5-3.7 ; P_{\text {trend }}=0.60$ ). Restricting analyses to clear cell RCC, or to cases with DNA extracted from buffy coat in PLCO or whole blood in USKC, or to USKC cases who had their blood sample collected within 12 months from diagnosis yielded virtually identical results (data not shown).

\begin{tabular}{|c|c|c|}
\hline & USKC & PLCO \\
\hline Characteristic & $N(\%)$ & $N(\%)$ \\
\hline $\begin{array}{l}\text { Age } \\
\quad \leqslant 59 \\
60-64 \\
65-69 \\
\geqslant 70\end{array}$ & $\begin{array}{r}355(51.9) \\
112(16.4) \\
97(14.2) \\
120(17.5) \\
\end{array}$ & $\begin{array}{l}70(29.0) \\
83(34.4) \\
55(22.8) \\
33(13.7) \\
\end{array}$ \\
\hline $\begin{array}{l}\text { Sex } \\
\text { Male } \\
\text { Female }\end{array}$ & $\begin{array}{l}390(57.0) \\
294(43.0)\end{array}$ & $\begin{array}{r}169(70.1) \\
72(29.9)\end{array}$ \\
\hline $\begin{array}{l}\text { Race } \\
\text { White } \\
\text { Black } \\
\text { Other }\end{array}$ & $\begin{array}{c}525(76.8) \\
159(23.2) \\
0(0.0)\end{array}$ & $\begin{array}{r}214 \text { (88.8) } \\
15(6.2) \\
12(5.0)\end{array}$ \\
\hline $\begin{array}{l}\text { Smoking status } \\
\text { Never } \\
\text { Past } \\
\text { Current }\end{array}$ & $\begin{array}{l}281(41.1) \\
234(34.2) \\
169(24.7)\end{array}$ & $\begin{array}{r}96(39.8) \\
122(50.6) \\
23(9.5)\end{array}$ \\
\hline $\begin{array}{l}\text { BMI }\left(\mathrm{kg} \mathrm{m}^{-\mathbf{2}}\right) \\
\quad<25 \\
25-29.9 \\
30-34.9 \\
\geqslant 35\end{array}$ & $\begin{array}{l}133(19.4) \\
239(34.9) \\
169(24.7) \\
143(20.9)\end{array}$ & $\begin{array}{r}49(20.3) \\
101(41.9) \\
65(27.0) \\
26(10.8)\end{array}$ \\
\hline $\begin{array}{l}\text { Subtype } \\
\text { Clear cell } \\
\text { Papillary } \\
\text { Chromophobe } \\
\text { Cystic } \\
\text { Other }\end{array}$ & $\begin{array}{c}501(73.2) \\
93(13.6) \\
42(6.1) \\
36(5.3) \\
12(1.8)\end{array}$ & \\
\hline $\begin{array}{l}\text { Tumour size } \\
\quad \leqslant 4 \mathrm{~cm} \\
4.1-7 \mathrm{~cm} \\
>7 \mathrm{~cm}\end{array}$ & $\begin{array}{l}352(51.5) \\
184(26.9) \\
148(21.6)\end{array}$ & \\
\hline \multirow[t]{2}{*}{$\begin{array}{l}\text { Stage } \\
\text { I } \\
\text { II } \\
\text { III and IV }\end{array}$} & $\begin{array}{r}473(69.2) \\
82(12.0) \\
129(18.9)\end{array}$ & \\
\hline & Mean (s.d.) & Mean (s.d.) \\
\hline Telomere length ( $\mathrm{T} / \mathrm{S}$ ratio) & $0.9(0.2)$ & $1.4(0.6)$ \\
\hline
\end{tabular}


Table 2. Association between leukocyte telomere length (LTL) and disease-specific survival among renal cell carcinoma patients from the US Kidney Cancer Study (USKC) and the prostate, lung, colorectal and ovarian cancer (PLCO) screening trial ${ }^{a}$

\begin{tabular}{|c|c|c|c|c|c|c|c|c|c|}
\hline \multirow[b]{3}{*}{ Study } & \multicolumn{9}{|c|}{ Quartile of LTL } \\
\hline & \multicolumn{2}{|c|}{ Q4 } & \multicolumn{2}{|c|}{ Q3 } & \multicolumn{2}{|c|}{ Q2 } & \multicolumn{2}{|c|}{ Q1 } & \multirow[b]{2}{*}{$P_{\text {trend }}$} \\
\hline & $n$ (event) & $\mathrm{HR}$ & n (event) & HR $(95 \% \mathrm{Cl})$ & n (event) & HR $(95 \% \mathrm{Cl})$ & n (event) & HR $(95 \% \mathrm{Cl})$ & \\
\hline $\begin{array}{l}\text { USKC } \\
\text { Model } 1^{b} \\
\text { Model } 2^{c}\end{array}$ & $171(20)$ & $\begin{array}{l}1.0 \\
1.0\end{array}$ & $171(24)$ & $\begin{array}{l}1.3(0.7-2.4) \\
1.5(0.7-3.0)\end{array}$ & $171(21)$ & $\begin{array}{l}1.1(0.6-2.0) \\
1.7(0.8-3.5)\end{array}$ & $171(31)$ & $\begin{array}{l}1.7(0.9-3.0) \\
2.3(1.2-4.4)\end{array}$ & $\begin{array}{l}0.13 \\
0.02\end{array}$ \\
\hline $\begin{array}{l}\mathrm{PLCO}^{\mathbf{d}} \\
\text { Years from blood draw } \\
\quad \leqslant 6.5 \\
>6.5\end{array}$ & $\begin{array}{l}60(15) \\
27(9) \\
33(6)\end{array}$ & $\begin{array}{l}1.0 \\
1.0 \\
1.0\end{array}$ & $\begin{array}{l}60(11) \\
19(4) \\
41(7)\end{array}$ & $\begin{array}{l}1.0(0.4-2.5) \\
0.6(0.1-2.4) \\
0.8(0.2-3.0)\end{array}$ & $\begin{array}{l}57(14) \\
32(9) \\
25(5)\end{array}$ & $\begin{array}{l}2.0(0.8-4.9) \\
1.9(0.5-7.8) \\
2.0(0.4-10.5)\end{array}$ & $\begin{array}{l}29(7) \\
35(15)\end{array}$ & $\begin{array}{l}2.4(1.0-5.4) \\
1.4(0.3-6.1) \\
3.4(0.9-13.2)\end{array}$ & $\begin{array}{l}0.75 \\
0.04\end{array}$ \\
\hline Combined $^{\mathbf{e}}$ & & 1.0 & & $1.2(0.7-2.0)$ & & $1.8(1.1-3.0)$ & & $2.3(1.3-4.1)$ & 0.002 \\
\hline \multicolumn{10}{|c|}{ 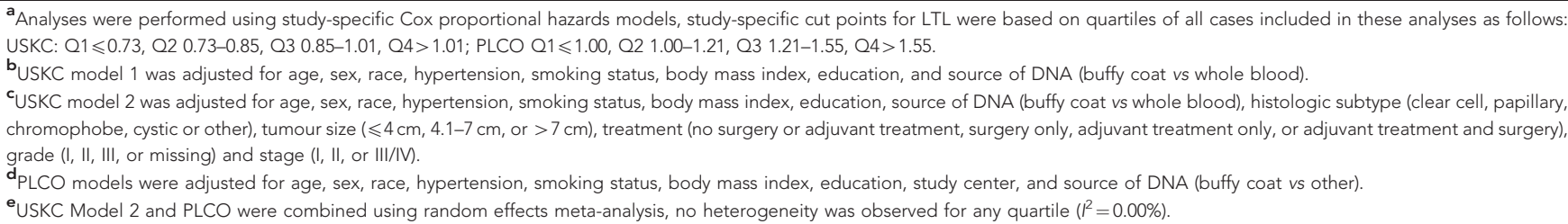 } \\
\hline
\end{tabular}

Table 3. Association between leukocyte telomere length (LTL), stage, and disease-specific survival among renal cell carcinoma patients from the US Kidney Cancer Study (USKC)

\begin{tabular}{|l|c|c|c|c|}
\hline Stage & $L T L$ & $\mathbf{n}$ (event) & HR $^{\mathbf{a}}(95 \% \mathrm{Cl})$ & P $_{\text {interaction }}{ }^{\mathbf{b}}$ \\
\hline $\mathrm{I}$ & $>0.85$ & $225(3)$ & 1.0 & 0.01 \\
\hline $\mathrm{I}$ & $\leqslant 0.85$ & $248(18)$ & $5.5(1.6-19.0)$ & \\
\hline $\mathrm{II}$ & $>0.85$ & $51(13)$ & $7.4(1.8-30.0)$ & \\
\hline $\mathrm{II}$ & $\leqslant 0.85$ & $31(9)$ & $9.0(2.1-38.7)$ & \\
\hline III/IV & $>0.85$ & $66(28)$ & $19.0(5.2-68.9)$ & \\
\hline III/IV & $\leqslant 0.85$ & $63(25)$ & $16.8(4.6-62.0)$ & \\
\hline
\end{tabular}

${ }^{a}$ Adjusted for age, sex, race, hypertension, smoking status, body mass index, education, source of DNA (buffy coat vs whole blood), histologic subtype (clear cell, papillary, chromophobe, cystic or other), tumour size $(<4 \mathrm{~cm}, 4.1-7 \mathrm{~cm}$, or $>7 \mathrm{~cm})$, grade $(\mathrm{I}, \mathrm{II}, \mathrm{III}$, or missing).

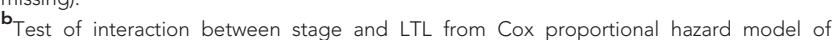
disease-specific survival.

In a joint analysis of stage and LTL, USKC cases with stage-I RCC and below-median $(\leqslant 0.85)$ LTL had significantly lower disease-specific survival than stage-I cases with LTL $>0.85$ (HR: 5.5, 95\% CI: 1.6-19.0; Table 3).

\section{DISCUSSION}

Our key finding, that shorter LTL is associated with poorer RCC disease-specific survival, is consistent with a prior study of 59 RCC cases with banked pre-diagnostic blood specimens and over 20 years of mortality follow-up, although the association in that study did not reach statistical significance (Weischer et al, 2013). Conversely, another study of 83 RCC cases including 22 deaths, using post-diagnostic samples, reported longer LTL to be associated with poorer prognosis; however, the association did not increase monotonically across quartiles of LTL (Svenson et al, 2009). Our study had a longer median follow-up time (USKC: 89 months PLCO: 168 months, vs 24 months) and a larger sample size than the study conducted by Svenson et al, which may, in part, explain our discordant findings. Interestingly, we found the association between LTL and survival to be strongest for stage-I RCC, which now accounts for most cases diagnosed in the United States (Kane et al, 2008).
The biologic basis for the relationship between telomere length and RCC survival is unclear. LTL may be a surrogate for telomere length in the renal proximal tubule, as qPCR measurements in peripheral blood leukocytes and non-malignant renal tissue are moderately correlated (Svenson et al, 2009). Critically shortened telomeres in renal epithelium or tumour cells may, through consequent genetic instability, be contributing to tumour progression (Hackett and Greider, 2002). Alternatively, short telomeres may be associated with acceleration of senescence of immune cells, which could lead to a reduced immune response and poorer RCC survival. For instance, in a study of patients with colorectal cancer those with shorter LTL had a lower percentage of B cells and circulating TGF$\beta 1$ (Chen et al, 2014). We note that aetiologic studies of LTL and RCC risk have yielded inconsistent findings (Wu et al, 2003; Shao et al, 2007; Hofmann et al, 2011, 2013; Weischer et al, 2013). In particular, one prior study of pre-diagnosis LTL that investigated both RCC risk and survival observed suggestive evidence of an association only for the latter end point (Weischer et al, 2013). Considering the evidence to date, it is thus unclear whether the biologic effects underlying measured short LTL play a role in driving the development of this malignancy.

A potential limitation of this investigation is that LTL was measured by different laboratories using different standard reference DNA, which resulted in different summary measures of LTL. To account for this issue, we used study-specific cut points and analysed the studies separately. The retrospective and prospective designs of these two studies are complementary to one another when considering potential sources of bias. For example, a potential limitation of the USKC study is the use of blood collected after diagnosis, raising the possibility that LTL alterations may have been caused by the disease or treatment. Reverse causation bias is suspected to be responsible for the typically stronger LTL associations reported from studies using post-diagnostic samples $v$ s prospective findings (Wentzensen et al, 2011). However, the confirmatory finding in PLCO cases, using pre-diagnostic blood, argues against such a bias. Conversely, the inability to adjust for stage and other clinical factors is a limitation of PLCO, while the USKC findings show the LTL-survival association is strengthened after adjustment for these factors (Table 2). Additional strengths of this investigation include its large size and the consistency in findings across studies.

Our observed association between short LTL and reduced RCC survival, particularly for stage-I disease, warrants replication in other 
study populations. If validated, this easily measured biomarker may have future clinical utility in staging of localised RCC.

\section{ACKNOWLEDGEMENTS}

This research was supported by the Intramural Research Program of the National Institutes of Health, National Cancer Institute, Division of Cancer Epidemiology, and Genetics.

\section{CONFLICT OF INTEREST}

RC has ownership interest (including patents) on method of measuring telomere length and is a consultant/advisory board member of Scientific Advisory Board, Telome Health, Inc. The remaining authors declare no conflict of interest.

\section{REFERENCES}

Chen Y, Qu F, He X, Bao G, Liu X, Wan S, Xing J (2014) Short leukocyte telomere length predicts poor prognosis and indicates altered immune functions in colorectal cancer patients. Ann Oncol 25(4): 869-876.

Hackett JA, Greider CW (2002) Balancing instability: dual roles for telomerase and telomere dysfunction in tumorigenesis. Oncogene 21(4): 619-626.

Hayes RB, Reding D, Kopp W, Subar AF, Bhat N, Rothman N, Caporaso N, Ziegler RG, Johnson CC, Weissfeld JL, Hoover RN, Hartge P, Palace C, Gohagan JK (2000) Etiologic and early marker studies in the prostate, lung, colorectal and ovarian (PLCO) cancer screening trial. Control Clin Trials 21(6 Suppl): 349s-355s.

Hofmann JN, Baccarelli A, Schwartz K, Davis FG, Ruterbusch JJ, Hoxha M, McCarthy BJ, Savage SA, Wacholder S, Rothman N, Graubard BI, Colt JS, Chow WH, Purdue MP (2011) Risk of renal cell carcinoma in relation to blood telomere length in a population-based case-control study. $\mathrm{Br} J$ Cancer 105(11): 1772-1775.

Hofmann JN, Lan Q, Cawthon R, Hosgood 3rd HD, Shuch B, Moore LE, Rothman N, Chow WH, Purdue MP (2013) A prospective study of leukocyte telomere length and risk of renal cell carcinoma. Cancer Epidemiol Biomarkers Prev 22(5): 997-1000.

Kane CJ, Mallin K, Ritchey J, Cooperberg MR, Carroll PR (2008) Renal cell cancer stage migration: analysis of the National Cancer Data Base. Cancer 113(1): $78-83$.

Schwartz K, Ruterbusch JJ, Colt JS, Miller DC, Chow WH, Purdue MP (2016) Racial disparities in overall survival among renal cell carcinoma patients with young age and small tumors. Cancer Med 5(2): 200-208.

Shao L, Wood CG, Zhang D, Tannir NM, Matin S, Dinney CP, Wu X (2007) Telomere dysfunction in peripheral lymphocytes as a potential predisposition factor for renal cancer. J Urol 178(4): 1492-1496.

Svenson U, Ljungberg B, Roos G (2009) Telomere length in peripheral blood predicts survival in clear cell renal cell carcinoma. Cancer Res 69(7): 2896-2901.

Weischer M, Nordestgaard BG, Cawthon RM, Freiberg JJ, Tybjaerg-Hansen A, Bojesen SE (2013) Short telomere length, cancer survival, and cancer risk in 47102 individuals. J Natl Cancer Inst 105(7): 459-468.

Wentzensen IM, Mirabello L, Pfeiffer RM, Savage SA (2011) The association of telomere length and cancer: a meta-analysis. Cancer Epidemiol Biomarkers Prev 20(6): 1238-1250.

Wu X, Amos CI, Zhu Y, Zhao H, Grossman BH, Shay JW, Luo S, Hong WK, Spitz MR (2003) Telomere dysfunction: a potential cancer predisposition factor. J Natl Cancer Inst 95(16): 1211-1218.

Zhang C, Chen X, Li L, Zhou Y, Wang C, Hou S (2015) The association between telomere length and cancer prognosis: evidence from a meta-analysis. PLoS One 10(7): e0133174.

This work is published under the standard license to publish agreement. After 12 months the work will become freely available and the license terms will switch to a Creative Commons AttributionNonCommercial-Share Alike 4.0 Unported License. 